\title{
Achieving precision in high density batch mode micro-electro-discharge machining
}

\author{
Mark T Richardson and Yogesh B Gianchandani \\ Department of Electrical Engineering and Computer Science, University of Michigan, \\ 1301 Beal Avenue, Ann Arbor, MI 48109, USA \\ E-mail: mtrichar@umich.edu
}

Received 11 August 2007, in final form 14 October 2007

Published 28 November 2007

Online at stacks.iop.org/JMM/18/015002

\begin{abstract}
This paper reports a parametric study of batch mode micro-electro-discharge machining $(\mu \mathrm{EDM})$ of high density features in stainless steel. Lithographically fabricated copper tools with single cross, parallel line and $8 \times 8$ circle/square array features of 5-100 $\mu \mathrm{m}$ width and 5-75 $\mu \mathrm{m}$ spacing were used to quantify trends in machining tolerance and the impact of debris accumulation. As the tool feature density is increased, debris accumulation effects begin to dominate, eventually degrading both tool and workpiece. Two independent techniques for mitigating this debris buildup are separately investigated. The first is a passivation coating which suppresses spurious discharges triggered from the sidewalls of the machining tool. By this method, the mean tool wear rate decreases from a typical of about $34 \%$ to $1.7 \%$ and machining non-uniformity reduces from $4.9 \mu \mathrm{m}$ to $1.1 \mu \mathrm{m}$ across the workpiece. The second technique involves a two-step machining process that enhances the hydrodynamic removal of machining debris compared to standard methods. This improves surface and edge finish, machining time and tool wear.
\end{abstract}

(Some figures in this article are in colour only in the electronic version)

\section{Introduction}

Micro-electro-discharge machining ( $\mu$ EDM) is a technique for patterning microstructures using controlled, sequential, spark discharges between a tool and workpiece of any conductive material $[1,2]$. These include non-traditional bulk materials that are typically difficult to machine such as stainless steel, tungsten carbide cobalt and permalloy. Feature sizes down to $5 \mu \mathrm{m}$ with a position accuracy of $0.1 \mu \mathrm{m}$ are possible.

Traditional $\mu \mathrm{EDM}$ is a serial process with limited throughput. A sharpened wire tip is scanned across the surface of the workpiece while immersed in a dielectric oil medium. An RC timing circuit controls the energy of each spark across a discharge gap of 1-3 $\mu \mathrm{m}$. Batch mode (die sinker) $\mu \mathrm{EDM}$ uses lithographically patterned, e.g. LIGA (lithographie galvanoformung abformung), electroplated copper, as cookiecutter-type tools to machine many features in parallel $[3,4]$. By utilizing multiple discharge circuits connected to segmented electrode arrays, batch mode can increase throughput by up to $100 \times$ over serial mode [5]. Applications for $\mu \mathrm{EDM}$ include the stentenna, injection molds, dc-to-dc boost conversion and radio frequency (RF) switches [6-9].
In terms of resolution and throughput, batch mode $\mu$ EDM complements other machining technologies such as computer numerical controlled (CNC) micro-milling, electrochemical etching, abrasive water jet and laser machining [10-14]. A common theme among this list is that they are all subtractive processes, allowing for a wider material selection than additive processes such as electroforming. Micromilling is susceptible to rapid tool wear when machining hard materials, and the overall performance is tied to the mechanical properties of the workpiece. Electro-chemical etching and abrasive water jet are fast processes, but have a limited resolution of around $\sim 70 \mu \mathrm{m}$. Laser machining can outperform $\mu \mathrm{EDM}$ in maximum resolution, but at the cost of machining speed since it is a serial process.

Micro-EDM is a thermal process similar to laser machining in that it leaves a recast layer on the surface after machining. Proper spark discharging is a complex sequence of events which is still being debated after more than 60 years [15-22] of use. The first stage after a sufficient voltage which is applied between the tool and the workpiece is electron migration, ionization and electron avalanching. Eventually a streamer (plasma channel) is formed between the electrodes 
allowing extremely high currents to pass, which primarily heat the anode (workpiece) side well above the melting point. When the current through the channel can no longer be sustained due to the RC timing circuit, the channel collapses. The violent implosion of the channel forces the molten spot in the workpiece into a crater formation, ejecting debris.

Understanding and exploiting these events have led to many improvements in machining performance. The pertinent steps for this work are the plasma channel initiation, plasma temperature and gas evolution. A point of contention among several studies is the precise mechanism for the plasma channel initiation. In water, one study points to the primary initiator as hydrogen bubbles from hydrolysis [18]. In oil, bubbles from hydrolysis do not occur as readily but debris particles seem to contribute. Regardless if this is the true mechanism, several studies have noted that a higher concentration of debris particles increases the likelihood of a discharge $[5,15,18]$.

In $[2,18,19]$, optical spectroscopy of the plasma shows that the electron temperature is on the order of $10000 \mathrm{~K}$. With metal contaminants, the temperature decreases to around $2700 \mathrm{~K}$ [18]. Residual debris particles from the tool and workpiece cause variation in discharge characteristics not only in location, but also in energy.

One point that may affect batch mode $\mu$ EDM much more than serial mode is gas evolution. For each spark discharge, a small amount of hydrogen gas in the plasma channel is left behind. In serial mode, this gas naturally coalesces into larger bubbles that eventually rise out of the discharge gap, entraining debris as they go [21, 22]. In batch mode, the tool areas are very large with only small separation from the workpiece. The bubbles tend to become trapped within the tool features. As noted before, gas bubbles could initiate discharges in water but if they are trapped in the discharge gap this may also be true in oil $[18,20]$.

Self-created machining debris is critical in determining tolerances for both serial mode and batch mode $\mu$ EDM [10]. In serial mode, the machining tool can be both rotated and vertically dithered to flush out debris. However, in batch mode, the tool movement is limited to a vertical dither motion. The large planar extent of the tool and its high-aspect ratio features limit traditional options for flushing.

When the density of features increases in batch mode, debris accumulation leads to spurious discharges that damage the workpiece surface and cause excessive tool wear [10, 23, 24]. Eventually, debris accumulation between the tool and the workpiece causes uncontrolled arcing, and stalls machining indefinitely. While the dielectric oil that separates workpiece from tool during machining does help dissipate heat, fluid flow is severely restricted in batch $\mu$ EDM. As machining progresses, local temperatures may increase. For batch mode $\mu$ EDM, copper electroplated into a photolithographically defined mold is a convenient choice. The benefits of copper are its high electrical and thermal conductivity as well as its process compatibility. While the melting point of copper is $>1000{ }^{\circ} \mathrm{C}$, it softens at much lower temperatures $\left(\sim 280{ }^{\circ} \mathrm{C}\right)$ [25]. The high local temperatures soften and recast the tool into a mushroom shape, which is then transferred to the workpiece. As the feature density increases, the problem limits both vertical cutting depth and lateral resolution.
Various methods for handling the debris buildup have been attempted. The main alternatives are adding vibration to the stage or tool [22, 26-33], integrating dielectric flushing to the stage or tool [34] and/or tool coatings [34, 35]. Stage vibration cyclically forces the tool toward the workpiece so that the dielectric oil and debris are pushed out of the discharge gap. The return stroke then pulls fresh oil into the gap. Applying a planetary motion to the tool improves the maximum aspect ratio but may also increases the minimum feature size $[22,26,27]$. Integrated dielectric flushing involves a nozzleshaped tool/workpiece that forces fresh dielectric oil into the discharge by positive or negative fluidic pressure [11]. Tool coating/encasing allows for a hybrid of material properties such as wear resistance and high cutting precision on a single tool [34]. While these methods have been applied to both macro- and microscale serial mode EDM, only a $\sim 10 \mu \mathrm{m}$ vertical or planetary stage vibration with frequencies in the $10 \mathrm{~s}$ of hertz has been reported for batch mode.

This paper ${ }^{1}$ examines scaling trends associated with debris generation in batch mode $\mu$ EDM on a Panasonic ED$72 \mu \mathrm{EDM}$ using a parametric study. By varying tool width and tool spacing, trends are found that point to improved designs. Two solutions for handling debris buildup are then presented. A passivation tool sidewall coating reduces spurious discharges and a two-step machining approach allows gas bubbles generated during machining to hydrodynamically flush debris away from the discharge gap.

\section{Parametric study}

Three different classes of features are presented in this study: isolated crosses, parallel lines and square arrays. All three are commonly used lithography patterns. Each feature adds a different complexity to the microfluidic path for debris to exit from the discharge gap.

\subsection{Tool fabrication}

Copper tools ranging from 175 to $200 \mu \mathrm{m}$ tall were fabricated at Sandia National Laboratories, CA using polymethylmethacrylate (PMMA) LIGA on a low-Z titanium oxide seed layer. The process begins with depositing the electroplating seed layer on a thick (1-2 mm) silicon wafer substrate and solvent bonding PMMA on top. X-ray exposure was done at the Advanced Light Source at Lawrence Berkeley National Laboratory. After developing the PMMA into a mold, copper was electroplated into it and lapped back to the finished thickness. After dicing, the PMMA was released in acetone.

These tools were used to machine patterns $30 \mu \mathrm{m}$ deep into $100 \mu \mathrm{m}$ thick, \#316L stainless steel foil workpieces with a Panasonic MG-ED72 $\mu$ EDM. The height of the tool did not influence the machining. The end of a $5 \mathrm{~mm}$ diameter aluminum mandrel was planarized using the WEDG process [36], and the workpiece was mounted to the mandrel using silver epoxy. The sample had a flatness of approximately $2 \mu \mathrm{m}$ after mounting.

1 Portions of this paper have appeared in a conference abstract form in $[23,24]$. 

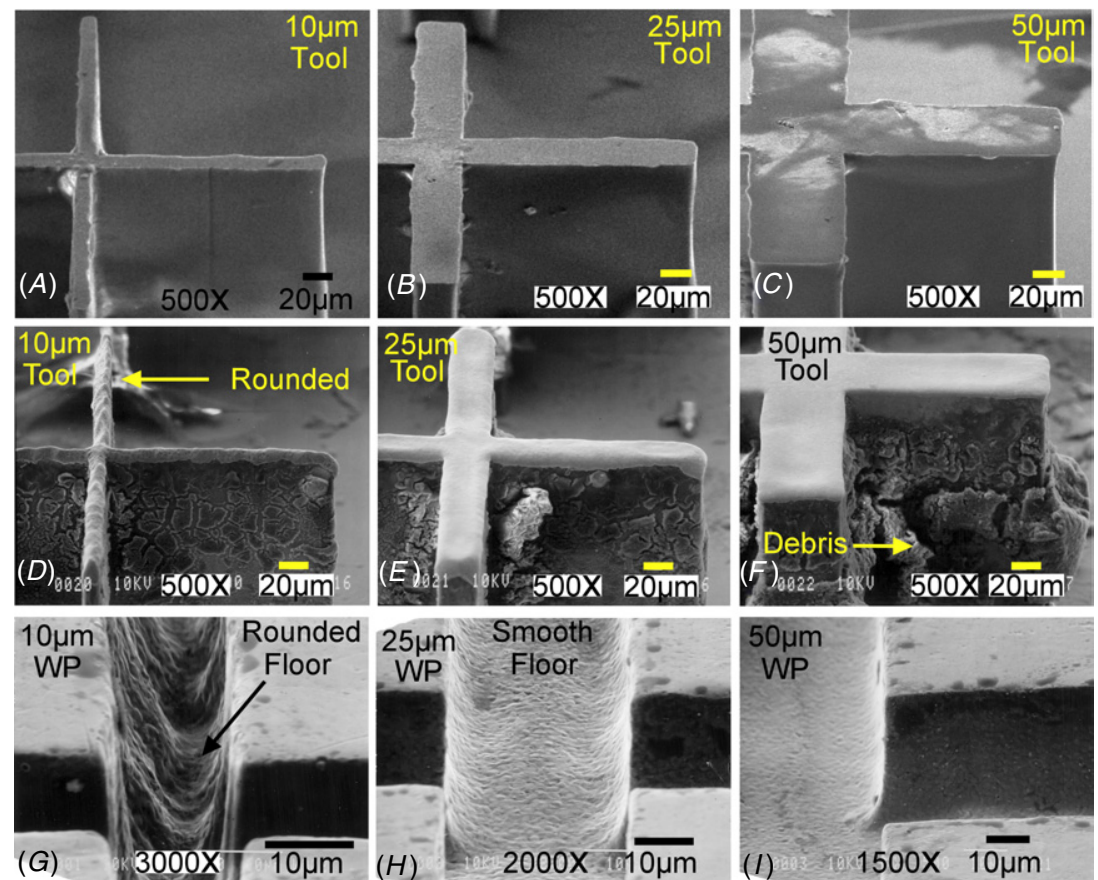

Figure 1. SEM image survey of $600 \mu \mathrm{m} \times 600 \mu \mathrm{m}$ cross features with varying widths of 10,25 and $50 \mu \mathrm{m}$. The top row $(A)-(C)$ shows freshly fabricated (unused) tools, the middle row shows tools that have been worn by the machining process and the bottom row shows machined workpiece features. Note the rounding of the tools after they have been used. Note the amount of debris along the sidewalls in $(F)$ compared to $(D)$ and $(E)$. $(G)$ shows effects of tool wear at the bottom of the workpiece. $(A)-(F)$ are at $500 \times$ magnification, while the remaining images are at higher magnification.

Table 1. Machining conditions for parametric study.

\begin{tabular}{ll}
\hline Voltage & $70 \mathrm{~V}$ \\
Capacitor & $10 \mathrm{pF}$ \\
Resistor & $5 \mathrm{k} \Omega$ \\
Z-feed & $0.2 \mu \mathrm{m} \mathrm{s}^{-1}$ \\
Stage dither & $\sim 10 \mu \mathrm{m}$ \\
\hline
\end{tabular}

\subsection{Experiments}

The isolated cross, parallel line and square array patterns used in this study each investigate different effects of debris on machining. Varying the size of a single cross shows the debris effects when the ratio of the machined surface area (debris volume) to the feature edge length (microfluidic resistance of the escape path) is changed. Parallel lines of varying width and spacing add the interaction of adjacent features to the escape path of the debris. Finally, a 2D array pattern of varying width and spacing adds another axis of interaction to the escape path.

Machining parameters for these experiments are given in table 1 and were chosen for surface smoothness and edge finish at the expense of machining time. Kerosene-based $\mu \mathrm{EDM}$ dielectric oil separated the tool from the workpiece during machining for heat dissipation, debris removal and discharge energy regulation. Electrical contact was made to the tool through the seed layer. Since the tool could not be rotated as in conventional serial EDM, the stage was dithered vertically to improve debris removal. While the dither amplitude and frequency were kept constant in this work, an investigation on the impact of varying these parameters to batch mode $\mu$ EDM performance would be useful.
Lateral dimensions at the top of tool and workpiece features were measured under high magnification on a calibrated optical microscope. SEM images showed that sidewalls were vertical with around $2-5 \mu \mathrm{m}$ of rounding at the bottom of the workpiece features that corresponded to tool wear rounding. Measurements of tool height and workpiece depth were taken by focusing a calibrated microscope on the top and then on the bottom surfaces. Tool plunge depth measurements during machining were recorded from the $\mu \mathrm{EDM}$ controller. It is important to note that this is simply the distance traveled from the first discharge and does not account for losses due to tool wear. Depth measurements were recorded at timed intervals, but the timing of erratic events was also recorded.

2.2.1. Crosses. The isolated feature study used $600 \mu \mathrm{m} \times$ $600 \mu \mathrm{m}$ long cross patterns to investigate the impact of varying tool widths, from 10 to $100 \mu \mathrm{m}$, on discharge gap due to selfgenerated debris. It also shows how machined feature edges vary in the middle of a cross arm compared to the end. All features were machined at once, ensuring the same conditions for comparison. Cross pattern tools were used because they are more mechanically robust during mold stripping compared to freestanding line patterns in the LIGA process. Prior to machining, the top surfaces were planar from lapping.

The SEMs of the tools and corresponding workpieces in figure 1 show that the $25 \mu \mathrm{m}$ tool provides a good compromise between good finish and low debris buildup. (The tool was not cleaned after machining, but the workpiece was cleaned in an ultrasonic bath.) In comparison, the $10 \mu \mathrm{m}$ tool had considerably more wear and rounding, which transferred to 


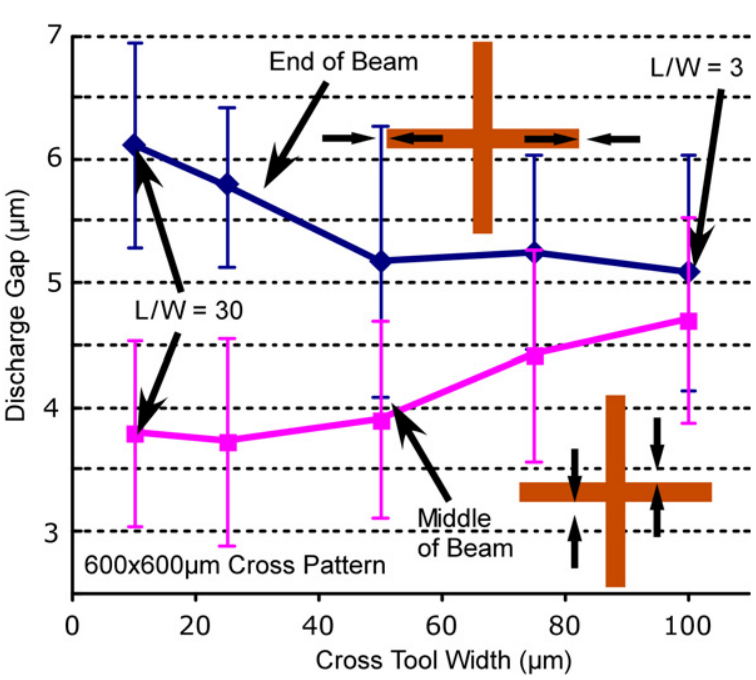

Figure 2. The discharge gap at the ends of the machined features (upper lines) were larger than at the center (lower line). However, for $600 \mu \mathrm{m} \times 600 \mu \mathrm{m}$ long features, as the feature widths change from $10 \mu \mathrm{m}$ to $100 \mu \mathrm{m}$, the discharge gaps at these two locations become comparable. Discharge gaps were determined by subtracting the tool width from the machined workpiece width and dividing by 2 . Error bars are 1 standard deviation from the mean $(n=20$ for each data point).

the workpiece. However, it also had very little residual debris. If the intent were to machine through the entire workpiece and the tool were much taller than the workpiece, this tool width could still be acceptable. The wider tools all show progressively better edge and surface finish albeit with some minor footing at the bottom of the workpiece and increasing residual debris. The images of the 75 and $100 \mu \mathrm{m}$ wide tools (not shown) were similar to the $50 \mu \mathrm{m}$ tool.

The discharge gap versus the tool width data for isolated crosses in figure 2 was taken using a filar eyepiece at $500 \times$ magnification. Each data point represents the subtraction of the mean initial tool dimension from the mean finished workpiece dimension, divided by $2(n=20)$ and the error bars are at \pm 1 standard deviation.
In the patterns that were tested, the tool feature width was varied, while the length of the features remained fixed. The ratio of the feature length to the feature width $(L / W)$ varied from 3 to 30. There are two distinct trends which depend on the region of the tool. The discharge gaps at the ends of the tools were generally larger than at the center. It was noted that when the $L / W$ ratio was small, the discharge gaps at the two locations became more similar; that is for a fixed length tool, as the feature width was increased the larger discharge gaps that were at the ends of the crosses tended to decrease in size, while the smaller discharge gaps that were near the middle of the crosses tended to increase in size (figure 2).

Fluent ${ }^{\mathrm{TM}}$ simulations were performed to determine if the hydraulic resistance for debris to escape could be related to these different trends in the discharge gap. Single, $300 \mu \mathrm{m}$ long lines (one cross leg) with variable widths were simulated. Eight fluidic pressures were applied at the inlet of a $30 \mu \mathrm{m}$ tall (channel length) annular rectangular channel and the integration of the flow at the outlet gave the total flow. The discharge gap (channel height) was $6 \mu \mathrm{m}$, viscosity $1.4 \mathrm{cP}$ and specific gravity 0.798 . Fluent ${ }^{\mathrm{TM}}$ uses the Navier Stokes equation and the continuity equation to solve the flow. Laminar flow with a no-slip boundary condition at the walls was assumed.

Figure 3(A) shows the simulation dimensions for a $10 \mu \mathrm{m}$ wide line tool feature. Figure $3(B)$ shows the flow profile at the inlet and outlet at $70 \mathrm{kPa}$. The corners form higher flow regions than the rest of the structure. The linear flow $(Q)$ versus pressure $(P)$ slope corresponds to the hydraulic resistance $(R)$ of the structure since $P=Q R$. As shown in the flow profile, the resistance is lower at the end of the line and more debris will likely tend to pass through that area. Since electrical discharges also tend to occur more frequently at edges and corners, this could lead to a local increase in spurious discharges and tool wear, which would account for the increase in discharge gap. For wider tools, the flow profile is more evenly distributed and tool wear decreases. In the midpoint of the line, the discharge gap likely increases with tool width because the amount of debris generated (tool surface
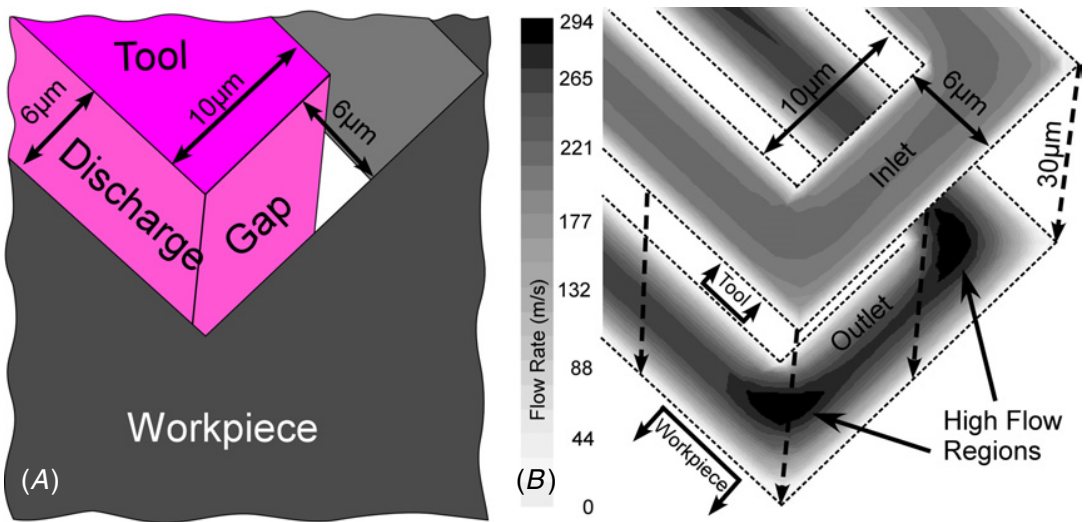

Figure 3. Fluent ${ }^{\mathrm{TM}}$ simulation of dielectric oil flow profile for discharge gap surrounding a $10 \times 300 \mu \mathrm{m}^{2}$ tool. (A) Simulation dimension definition. $(B)$ High flow region occurs at the sharp corners and on the edge connecting the corners. Increased debris flow in this region may lead to more spurious discharges that cause an increase in discharge gap. 


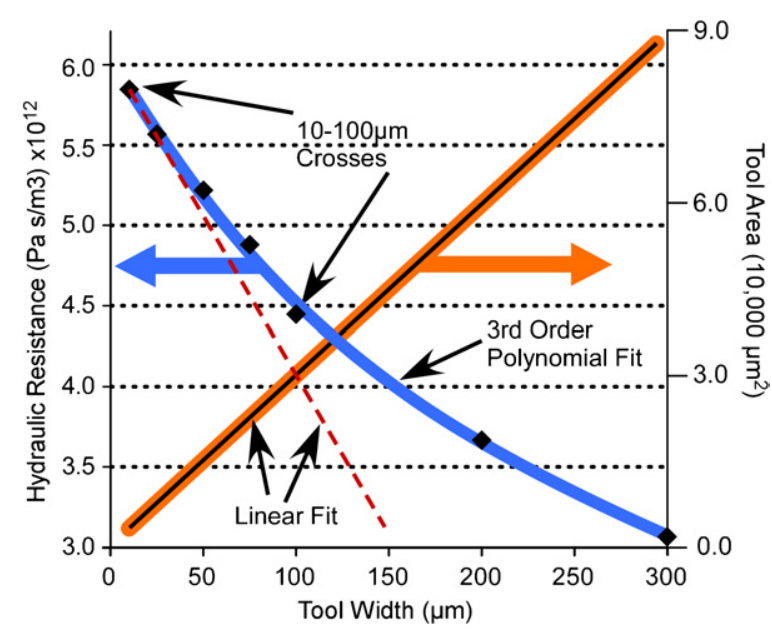

Figure 4. Discharge gap hydraulic resistance of a single line tool with a fixed length but variable width calculated with Fluent ${ }^{\mathrm{TM}}$ simulations. The area of the tool increases linearly with tool width, but the hydraulic resistance does not decrease linearly.

area) has increased while the local hydraulic resistance has remained nearly the same.

Figure 4 shows that as the tool width increases, the area for machining increases linearly while the hydraulic resistance of the channel surrounding the tool does not decrease linearly. This suggests that the path for the debris to escape may not be widening as fast as the amount of debris generated is increasing. As more debris accumulates in the discharge gap, it will likely widen to accommodate. Note that only the resistance on the side of the tool is accounted for in this model. As the width of the tool increases, the average distance from the actual discharge site to the side of the tool also increases.

2.2.2. Parallel line. The dimensional tolerance of grouped batch $\mu \mathrm{EDM}$ features was evaluated using $600 \mu \mathrm{m}$ long parallel trenches machined by four parallel lines of 10,15 , 25 and $50 \mu \mathrm{m}$ width and 5,10,15, 18, 20, 25 and $50 \mu \mathrm{m}$ spacing. The layout allowed for machining of 16 different sets of features at once while minimizing debris movement among different size features.

Parallel lines, $600 \mu \mathrm{m}$ long, with varying widths and spacing were machined with the same conditions as the crosses. Digital imaging was used to measure the width of the tool and workpiece features and compared with a known reference before and after machining. Figure 5 shows the machining tolerance for this test pattern. Machining tolerance is defined as the original tool feature spacing minus the actual machined width. This value is the addition of one discharge gap each from two adjacent line features. Three tool feature spacings are shown for clarity in figure 5 . While $5 \mu \mathrm{m}$ wide features can be machined, it is notable that the tolerances range from 5 to $17 \mu \mathrm{m}$ and have several trends.

Similar to cross features, the debris generation rate of a particular tool feature width does not scale at the same rate as the hydraulic resistance for the path to remove the debris. For these parallel lines, the small spacing between features forms an additional rectangular channel hydraulic resistance in series

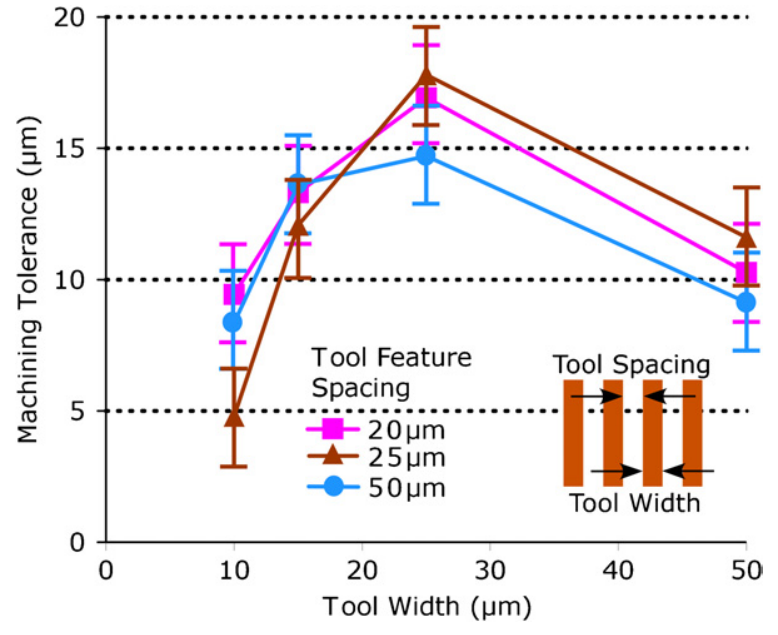

Figure 5. Machining tolerance (i.e. original tool feature spacing minus machined width) shows a nonlinear dependence on tool feature width and ranges from 5 to $17 \mu \mathrm{m}$. It indicates the necessary tool spacing to achieve a desired workpiece feature. Machining tolerance is the sum of two discharge gaps from adjacent features, whereas discharge gap measurements are taken from the same feature. Error bars are an estimate of the error in the measurement technique $(n=3)$.

with the discharge gap resistance and is defined by [37]

$$
R=\frac{12 \eta L}{H^{3} W-\frac{192}{\pi^{5}} H^{4} \sum_{m=0}^{\infty}(2 m+1)^{-5} \tanh \left[\frac{(2 m+1) \pi W}{2 H}\right]},
$$

where $\eta, L, H$ and $W$ are viscosity, length, height and width respectively. At the mid- point of the tool, for $20 \mu \mathrm{m}$ spacing, $175 \mu \mathrm{m}$ tall tool and $m=500$, this resistance is $2.24 \times$ $10^{12}(\mathrm{~Pa} \mathrm{~s}) \mathrm{m}^{-3}$ (43\% of the discharge gap resistance for $50 \mu \mathrm{m}$ tool width). The discharge gap separating the sidewall of the tool and the workpiece is strongly influenced by the width of the tool, but is different from the single cross feature in the previous section. The 20, 25 and $50 \mu \mathrm{m}$ feature spacing data follow the same trend, but there may be further trends. At a constant tool width of $25 \mu \mathrm{m}$, as the tool spacing increased, the machining tolerance improved.

For narrow tool features, the debris generation rate is low, the features are easily flushed when reasonably spaced, but the tool wear is high. As the tool feature widens, more debris is generated and the machined width increases more. Since the debris removal rate may not scale at the same pace, the debris cannot exit the discharge gap fast enough. Debris from adjacent features makes the problem even worse, leading to excessive tool wear.

2.2.3. Arrays. Arrays of features add another dimension of influence to the analysis. Squares were fabricated in $8 \times 8$ arrays with diameters ranging from 20 to $100 \mu \mathrm{m}$. In past work, it was found that the discharge gap was larger and the tool wear was greater at the center of an array $[4,10]$. In this study, the impact of tool diameter was concomitantly investigated. Inter-feature spacing was kept equal to the diameter because the impact of feature proximity was already investigated in the parallel trench study and may 


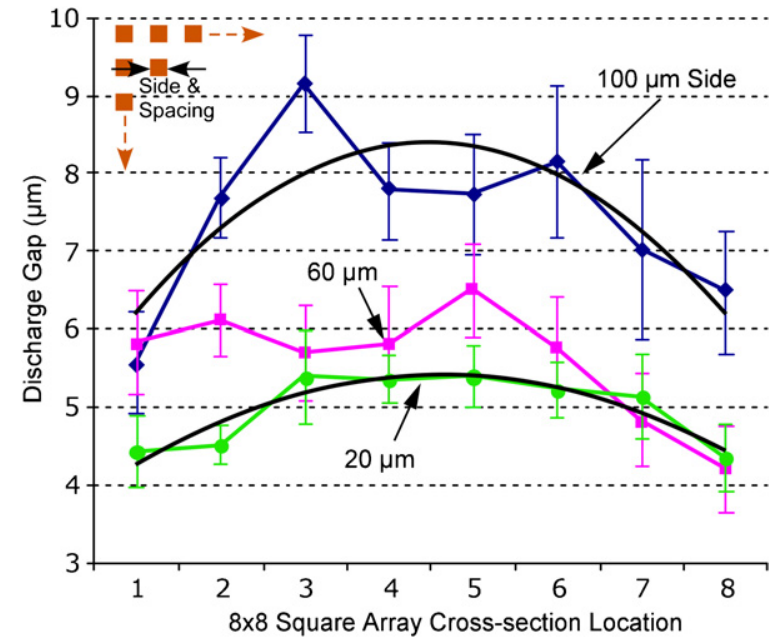

Figure 6. Discharge gap versus location along the diagonal of $8 \times$ 8 arrays with 20,60, $100 \mu \mathrm{m}$ side features and corresponding 20 , $60,100 \mu \mathrm{m}$ feature spacing. The discharge gap is larger in the center for all feature sizes. This effect is more pronounced as the feature size increases, even though feature spacing also increases at the same rate. The error bars are one standard deviation from the mean $(n=10)$.

have confounded the results. For square arrays, this means a $25 \%$ feature fill. The arrays were machined $35 \mu \mathrm{m}$ deep.

The discharge gap of each feature across the diagonal of every array was measured using a digital filar eyepiece (figure 6). There are two trends that should be noted. The first is that the discharge gap increases with increasing tool diameter, in agreement with the cross feature experiments. The second is that the discharge gap increases in the center of each array, also in agreement with past work [4]. Further, comparing the trend lines, the interior gap trend is shown to be even stronger as the tool diameter increases.

\subsection{Parametric study conclusion}

The parametric study presented above demonstrated that debris accumulation in high density batch $\mu \mathrm{EDM}$ can constrain precision in a significant way. In the single cross features, debris effects caused uneven tool wear. As features were packed closer and closer together in the parallel line features, debris effects increased. Finally, in the array study, it was shown that the non-uniform tool wear becomes worse as the size of the global pattern increases, even with the same fill factor. The following sections demonstrate two solutions for handling debris accumulation in high density batch $\mu \mathrm{EDM}$.

\section{Passivation coating}

\subsection{Design}

During $\mu \mathrm{EDM}$ machining, the presence of debris in the discharge gap lowers the threshold to initiate a discharge, causing spurious discharges on the sidewalls and base of the tool $[10,18]$. Protection from spurious discharges is especially important for high density patterns where debris tends to accumulate quickly. Uncontrolled debris accumulation can eventually cause heating and recasting of the features resulting in a mushroom shape. The new shape compromises not only the resolution of machined features, but also the tool lifetime. This phenomenon, previously reported in [10], is illustrated in figure 7(A) for the batch $\mu \mathrm{EDM}$ context.

Another factor is that a conductive particle can transport charge from one electrode to another and discharge at the opposite electrode [38]. When this occurs in $\mu \mathrm{EDM}$, debris

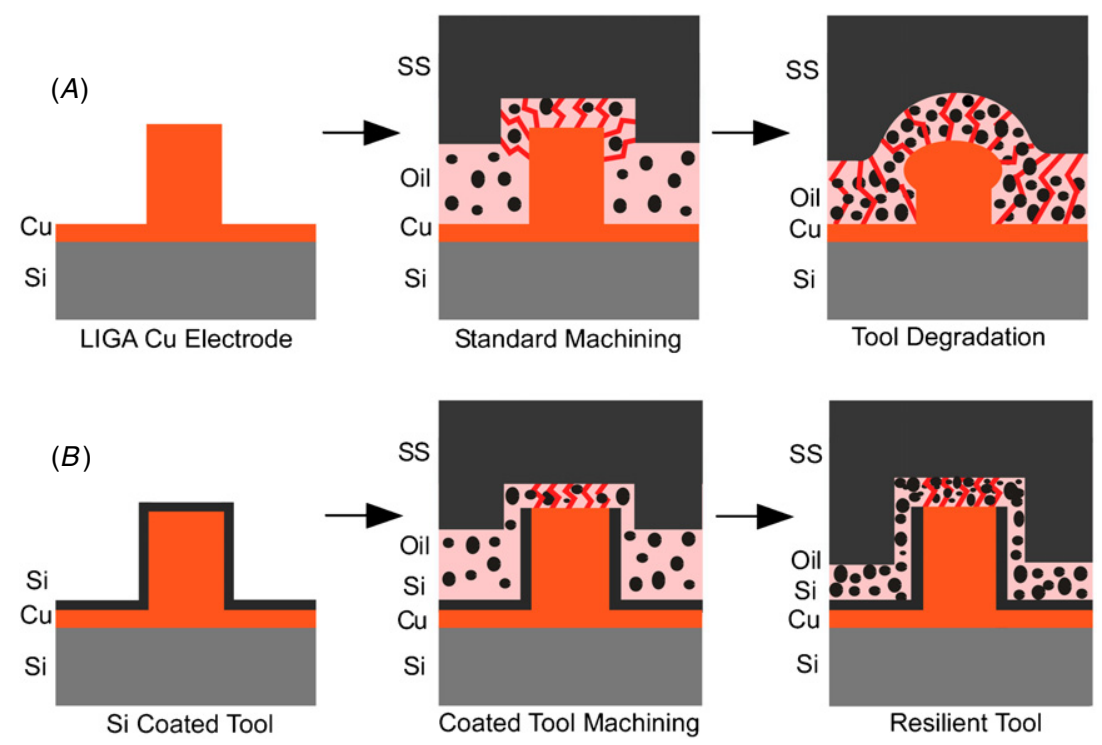

Figure 7. (A) A comparison of machining progression for uncoated tool and (B) passivation coated tool. The coating is selectively removed from the top of the tool by $\mu \mathrm{EDM}$ against a planar surface. Rounded workpiece edges and melted tool result for the uncoated case and selective machining for the coated case. 
particles generated during machining carry charge from the tool to the surface of the workpiece. The particles then discharge and damage the surface and edges of the workpiece. As debris accumulates between the workpiece and tool, these discharges occur more and more frequently along with normal discharges. Eventually, a conductive bridge is formed that increases heat generation dramatically. Not only can this condition stall machining indefinitely, but it can also increase the discharge crater size by an order of magnitude and cause local welding of debris to both workpiece and tool [10, 39]. The plunge depth at which this stalling occurred for the control pattern used in this study was approximately $25 \mu \mathrm{m}$.

One way to improve machining is to add an insulating layer on the tool sidewalls to decrease spurious discharges (figure 7(B)). In [34], for macroscale serial EDM, a PTFE tube surrounding the tool electrode was used with mixed results. In this study an insulating coating is integrated directly on a lithographically fabricated tool for batch $\mu \mathrm{EDM}$. The purpose of a tool passivation coating is to provide protection from spurious discharges on the side and bottom surfaces of the tool by locally increasing the series resistance for spurious discharges (figure $7(B)$ ). The primary requirements for this coating are a thin film that is conformal, insulating and selectively removable at the top surface of the tool. Sputtered silicon was chosen for the coating material due to its relatively high resistivity, thermal conductivity and hardness. At small thicknesses, it is also conductive enough to be selectively removed with $\mu \mathrm{EDM}$ by reversing the machining voltage polarity as the tool surface is brought to a flat workpiece. Then, when machining in normal polarity, discharges will occur preferentially at the top of the (copper) tool which offers the path of least resistance.

Silicon has a higher melting point than copper and will not readily soften whereas copper softens at $280^{\circ} \mathrm{C}$ [25]. While silicon and copper do form a eutectic around $650{ }^{\circ} \mathrm{C}$ [40], this is much higher than the softening temperature of copper. The thin coating allows heat exchange from the copper to the oil, but restricts heat generation to only the top surface of the tool where the discharges occur. A silicon coating may also help maintain tool edge resolution by preventing the copper from recasting over the sides. As pattern density increases and/or tool height decreases, even more limited fluid flow would further exacerbate these problems.

\subsection{Fabrication}

An SU-8 UV LIGA process was used to fabricate $80 \mu \mathrm{m}$ tall copper tools with $10 \mu \mathrm{m}$ features. Since the sidewalls were not required to be precisely vertical, SU-8 UV LIGA provided a very economical and fast solution for high-aspect ratio molds compared to X-ray LIGA. SU-8 2025 with various adhesion layers [9] and a copper seed was used as a mold for copper sulfate electroplating. After lapping to planarize the top surface, a CF4 and O2 RIE plasma was used to strip the SU-8, yielding the control experiment structure in figure $7(A)$. The $2 \mathrm{~cm} \times 2 \mathrm{~cm}$ dies were then sputtered with a $500 \AA$ titanium adhesion layer and either $1000 \AA$ or $2000 \AA$ of silicon as in figure $7(B)$. From a processing perspective, silicon was

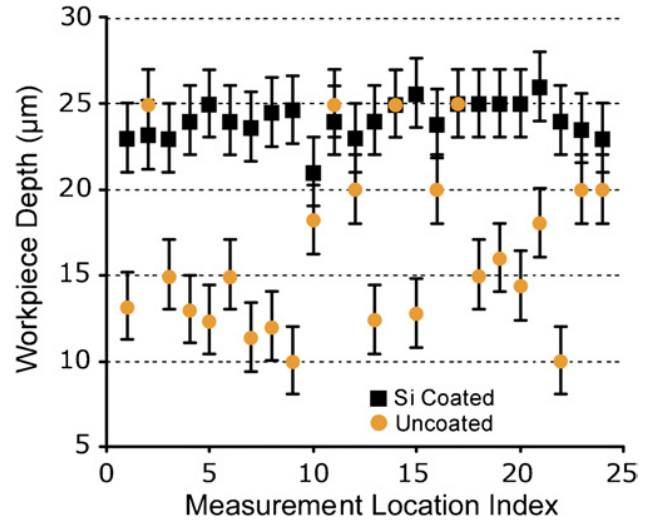

Figure 8. A comparison of machined workpiece depth at several locations across test pattern after plunging the tool $25 \mu \mathrm{m}$. The uncoated workpiece did not machine the full $25 \mu \mathrm{m}$ in most areas and also varied excessively across the pattern compared to the coated case. This difference in machined depth would account for the faster machining time for the uncoated case. Error bars are an estimate of the error in the measurement technique $(n=5)$.

Table 2. Machining conditions for coating $\mu \mathrm{EDM}$.

\begin{tabular}{llc}
\hline & Coating removal & Machining \\
\hline Voltage $(\mathrm{VDC})$ & $90-100$ & -70 \\
Z-feed $\left(\mu \mathrm{m} \mathrm{s}^{-1}\right)$ & 0.4 & 0.4 \\
Stage dither $(\mu \mathrm{m})$ & None & $\sim 10$ \\
Plunge $(\mu \mathrm{m})$ & 2 & 25 \\
\hline
\end{tabular}

chosen for the coating material since it is very resistive but still conducts enough to be electro-discharge machined at high voltages. As the copper tool wears down (slower than normal), the silicon coating wears down as well. The die with $2000 \AA$ of $\mathrm{Si}$ was used since the coating proved machinable and was more resistive.

A 250-350 $\mu \mathrm{m}$ thick $7 \mathrm{~mm} \times 6 \mathrm{~mm} \# 304$ stainless steel workpiece cut from a foil was then mounted to the mandrel using silver epoxy. Electrical contact was made to the tool directly on the copper seed in an area which was not coated. Setting the voltage of the coated tool to act as the workpiece, the silicon and titanium on the top surface of the tool were machined off with the stainless steel. In a new area of steel, the polarity was reversed and the tool plunged $25 \mu \mathrm{m}$ into the steel. Machining parameters are listed in table 2 and were chosen to minimize discharge energy, sacrificing machining time. The silicon-coated tool preferentially machined at the top since the resistive path was significantly lower there. The test pattern was similar to that used in an RF switch [9]. The same pattern was machined using a tool with the coating and a tool without the coating.

\subsection{Results}

For the uncoated tool, the time to reach a plunge depth of $25 \mu \mathrm{m}$ was $34 \mathrm{~min}$ and $3 \mathrm{~h}$, and $18 \mathrm{~min}$ for the coated tool. The uncoated machining (plunge depth) progressed steadily with only minor pauses until reaching the $25 \mu \mathrm{m}$ mark [23]. The coated machining on the other hand stalled for about $45 \mathrm{~min}$ before progressing at a slower rate past $10 \mu \mathrm{m}$. A stall is 

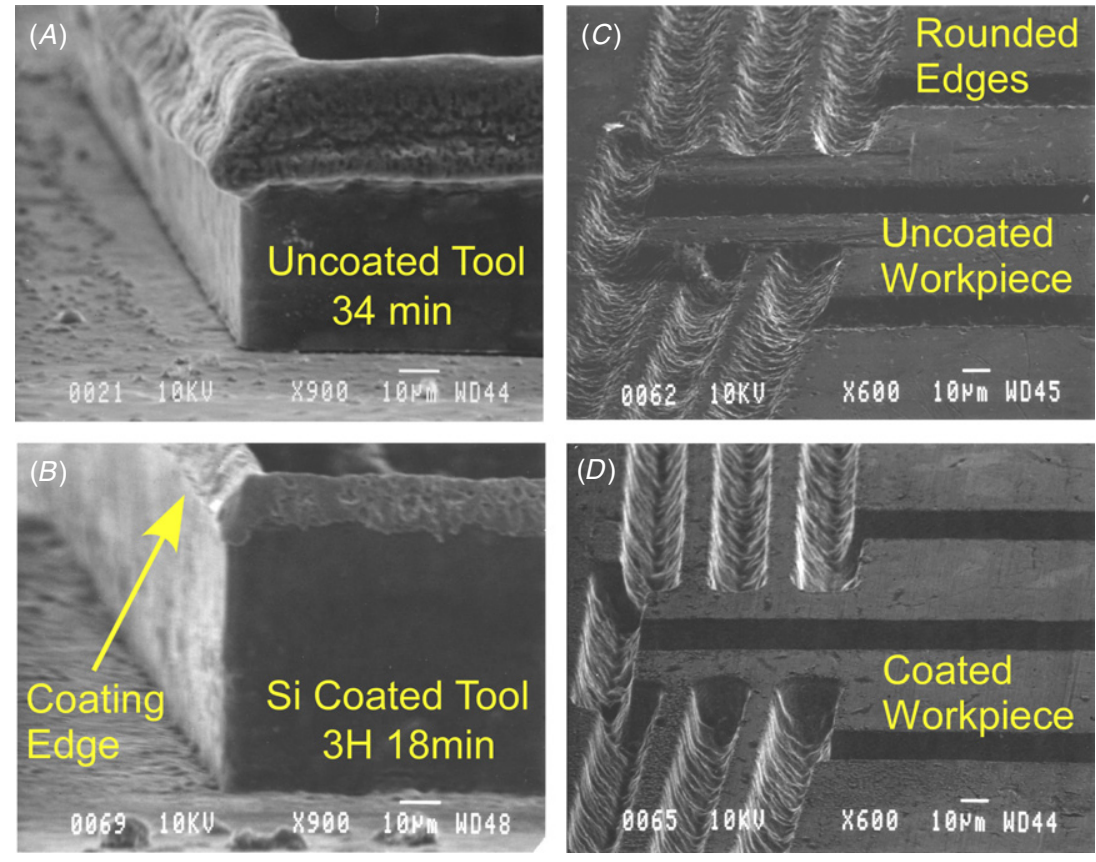

Figure 9. (A) Uncoated tool recasts into a mushroom shape after machining. (B) Silicon coated tool has negligible wear. $(C)$ Uncoated workpiece with feature rounding, edge rounding and rough surfaces. $(D)$ Coated workpiece with sharp features, sharp edges especially on corners, better surface finish and much deeper machining.

defined as the EDM control unit cyclically separating the tool from the workpiece, because a short circuit was detected, and then attempting to resume machining. While the uncoated tool machined faster, this result is misleading. As will be described, the uncoated tool destroyed itself as it machined and did not actually achieve the plunge depth. The coated tool did and remained intact.

Figure 8 shows data on the actual workpiece machining depth for various locations. Each data point represents the average of five measurements. The mean actual machined depth for the uncoated tool was $16.6 \mu \mathrm{m}$ while the coated tool was $24.1 \mu \mathrm{m}$. This corresponds to depth errors of $33.6 \%$ and $3.6 \%$, respectively, for an expected depth of $25 \mu \mathrm{m}$. This large difference can explain why the uncoated machining reached the target plunge depth so quickly. The uncoated tool was not machining the entire depth. The depth of machining was also more uniform for the coated case. A standard deviation of $4.9 \mu \mathrm{m}$ was calculated for the uncoated case compared to $1.1 \mu \mathrm{m}$ for the coated, which is a $78 \%$ improvement.

Figures $9(A)$ and $(B)$ show typical $80 \mu \mathrm{m}$ tall tool features after plunging $25 \mu \mathrm{m}$. The mushroom shape at the top of the tool is believed to have caused rounding of the workpiece edges in figure $9(C)$. The workpiece from the coated machining in figure $9(D)$ has very sharp edges, smoother surfaces and is visibly deeper. The mean control tool wear across all data points was $5.7 \mu \mathrm{m}$ for the uncoated tool compared to $0.4 \mu \mathrm{m}$ for the coated tool [23]. The tool wear rate is the mean tool wear divided by the mean actual machined depth. The uncoated case had a typical tool wear rate of $34 \%$ and the coated case had a typical rate of $1.7 \%$ despite machining significantly deeper and longer.

\subsection{Discussion}

As feature density increases, debris accumulation becomes a major concern because there is no efficient mechanism for removal. Machining took longer with the coated tools, presumably because debris accumulated in the discharge gap is slowly pulverized and flushed away. The EDM controller repeatedly detected short circuits and backed the tool out completely before progressing slowly to remove the blockage. Spurious discharges on the sidewalls may in fact provide a mechanism to break down debris into finer pieces and facilitate better flushing. In uncoated tools, the mushrooming may have also allowed for comparatively more efficient debris removal by limiting the actual depth of machining. However, the faster machining time in these tools comes at the cost of surface quality, machining depth, depth uniformity and tool wear. At even greater machining depths, debris accumulation and mushroom-shaped recasting exacerbate the problems further. A silicon passivation coating preliminarily addresses these problems. By combining a passivation coating with an integrated flushing technique, described next, a more complete solution for batch mode $\mu \mathrm{EDM}$ emerges.

\section{Hydrodynamic through-hole flushing}

A two-step machining method facilitates hydrodynamic removal of debris. By first $\mu$ EDM-machining narrow throughholes in the workpiece, a path is created for the debris to escape when the second, overlaid finishing pattern, is machined (figure 10). Self-generated bubbles from the discharge process entrain debris particles and carry them away from the discharge gap $[22,26]$. 


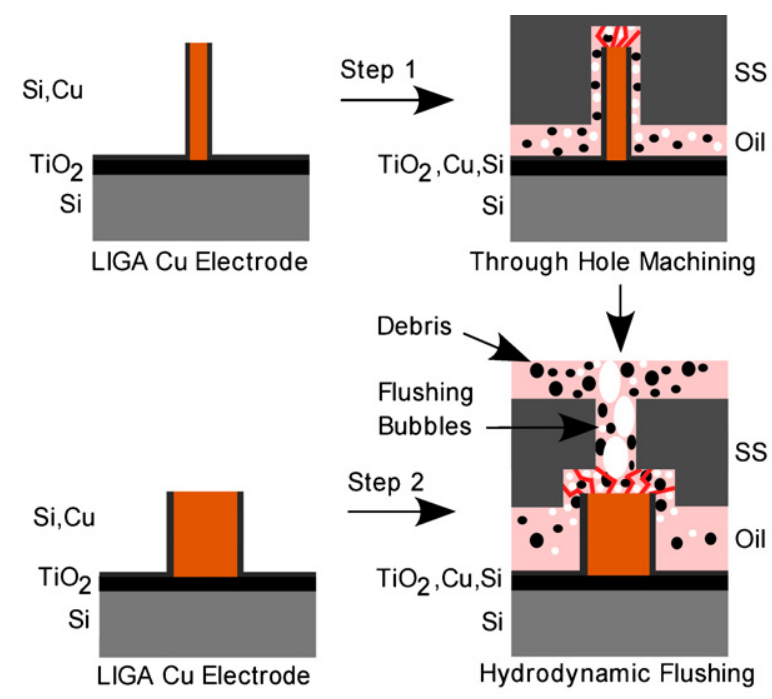

Figure 10. Machining process for two-step hydrodynamic debris flushing. Step 1: machine narrow through-holes, step 2: overlay workpiece on the new tool area and machine finished pattern. Flushing bubbles and tool dither force debris out the through-hole.

Table 3. Debris study machining conditions.

\begin{tabular}{ll}
\hline Tool height & $175 \mu \mathrm{m}$ \\
Finish hole & $120 \times 120 \mu \mathrm{m}^{2}$ \\
Wall thickness & $40 \mu \mathrm{m}$ \\
Wall spacing & $120 \mu \mathrm{m}$ \\
Z-feed & $0.2 \mu \mathrm{m} \mathrm{s}^{-1}$ \\
Plunge & $41,80 \mu \mathrm{m}$ \\
\hline
\end{tabular}

\subsection{Tool design and fabrication}

The control experiment pattern consisted of four large holes within an enclosed perimeter to represent structures with large machining fill factors. These patterns are resistant to flushing by the normal vertical tool dither in dielectric oil (table 3 ). In these types of patterns, debris generated by $\mu \mathrm{EDM}$ accumulates within the perimeter and forms conductive paths to the workpiece, causing machining to stall indefinitely.

In the two-step design, locating the through-holes in the field region of the final die does not disrupt the finished pattern. In this study, the through-holes were formed by $60 \mu \mathrm{m} \times 60 \mu \mathrm{m}$ posts located on the tool die next to the actual pattern. Thus, the workpiece was machined sequentially by two different parts of the tool die in separate steps.

Copper tools ranging from 175 to $200 \mu \mathrm{m}$ tall for both control and two-step patterns were fabricated with the process described in the section 2. A 2000 A silicon passivation coating was used as described in the previous section. These tools were used to machine patterns part way into $100 \mu \mathrm{m} 316 \mathrm{~L}$ stainless steel using the same machining conditions from the parametric study.

\subsection{Modeling}

The performance of the hydrodynamic debris removal technique cannot readily be fully modeled since it is a complex three-phase flow with changing boundary conditions.
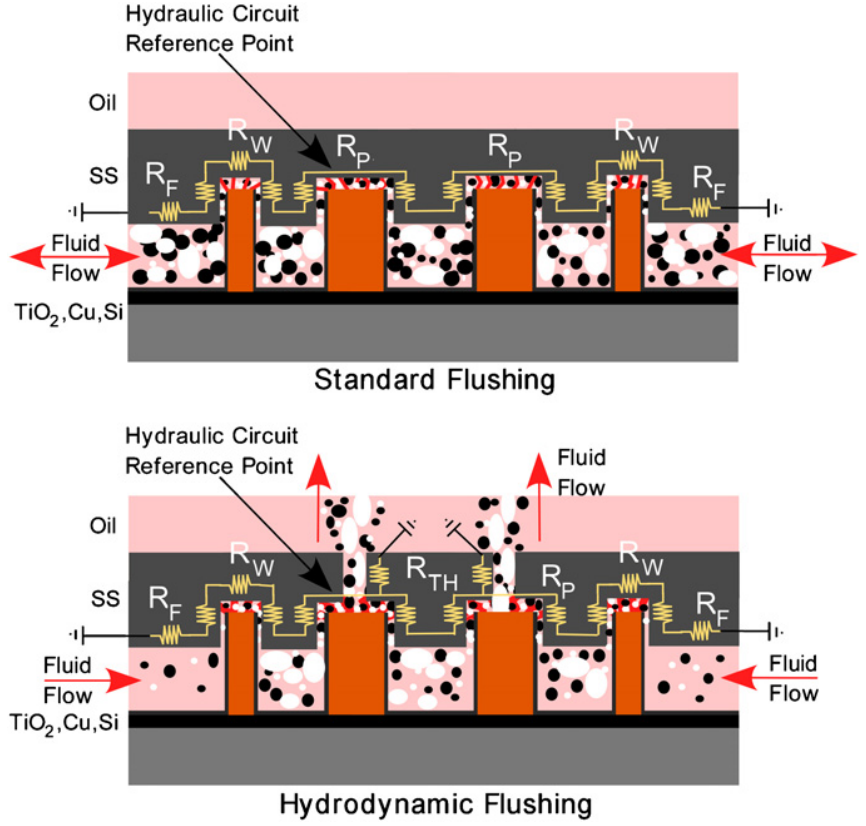

Figure 11. Cross section of hydraulic resistance circuit for standard dither flushing (top) and hydrodynamic flushing (bottom). Through-holes provide a shunt path for debris to escape by bubble entrainment.

Gas and debris particulate generation occurs at unknown rates. Significant but localized fluctuations in temperature and pressure fluctuations result from the spark discharges. The vertical dither of the tool also complicates the model. The standard debris removal mechanism involves fluid flow from the vertical tool dither and gas bubble entrainment. These effects are difficult to quantify with observation in batch mode. However, by assuming laminar flow, we can compare the hydraulic resistance at the locations of debris generation for a single point in time to gain some qualitative insight on the removal rates of the standard mechanism and the hydrodynamic mechanism.

For laminar flow, the hydraulic resistance of a rectangular channel is given by equation (1). For a preliminary comparison it is sufficient to examine the relative impact on an equivalent uniaxial hydraulic resistance. A cross section of the hydraulic circuit for the enclosed perimeter is shown in figure 11 and the full pattern is shown in figures $12(C),(D)$. The point of reference for the model is at an inner post with a plunge depth of $40 \mu \mathrm{m}$ and a discharge gap (channel height $H$ ) of $6 \mu \mathrm{m}$. To simplify the calculation for $R_{\text {Wall }}$, the outer wall can be represented as a rectangle of width $W$ and flow channel length $L_{\text {Wall }}$. Here, $W$ is the perimeter of the feature whereas

$$
L_{\text {Wall }}=2 \times(\text { plunge_depth })+(\text { wall_width }) .
$$

A similar method is used for $R_{\text {Post }}$ and $R_{\text {Field. }}$. Note that this equivalent width approach for square annular hydraulic resistance is about $36 \%$ too low compared to the Fluent ${ }^{\mathrm{TM}}$ simulations from the parametric study.) Therefore, these calculations can be easily compensated to obtain good accuracy with much less simulation time. Since the area of the workpiece field is much larger than the machined feature, 

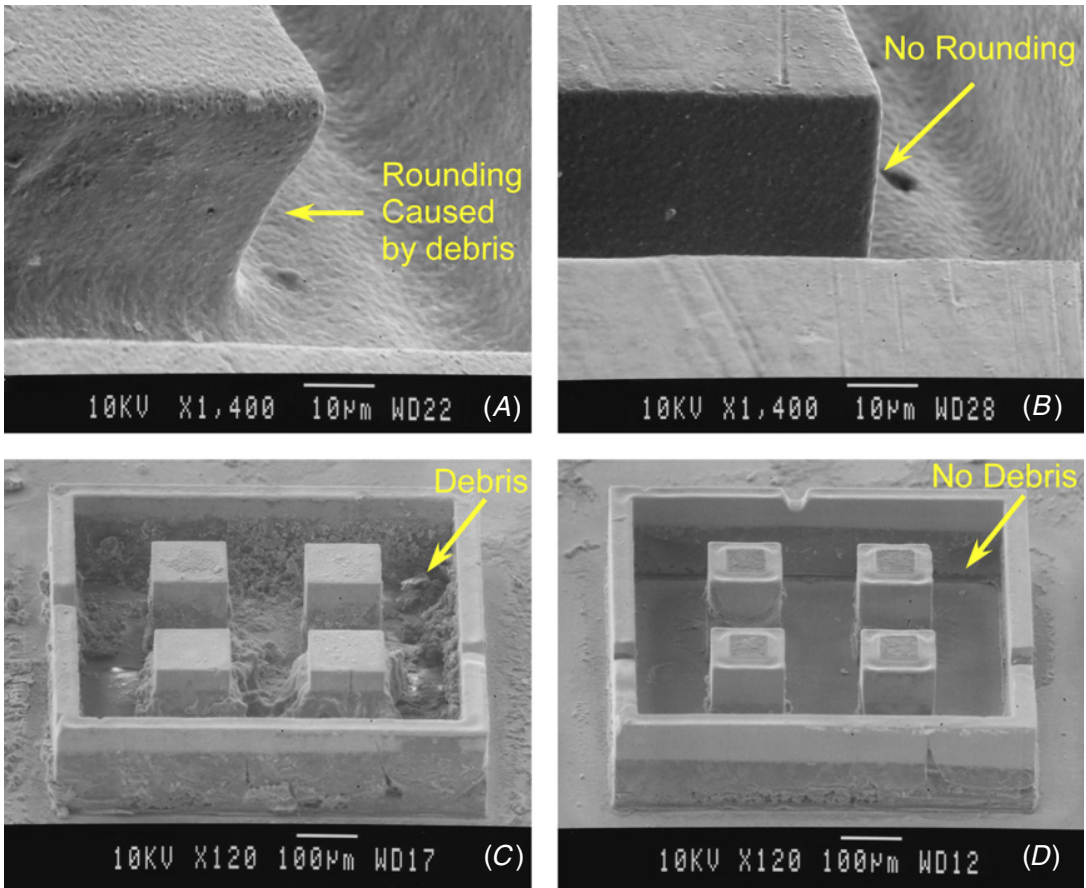

Figure 12. $(A)-(D)$ Enclosed workpiece (41 $\mu \mathrm{m}$ deep, 1 h $43 \mathrm{~min})(A)$ with no flushing. Rough surface on top and sidewalls, angled corner edge. Flushing workpiece ( $80 \mu \mathrm{m}$ deep, $1 \mathrm{~h} 59 \mathrm{~min})(B)$ shows clean sidewall and top surface, sharp corner. The grain structure is still visible on the top surface. Enclosed tool feature after machining $(C)$ has a lot of residual debris while flushing tool feature $(D)$ does not. Both utilized Si coated sidewalls.

a channel length of $2 \mathrm{~mm}$ is assumed for $R_{\text {Field. }}$ The total hydraulic resistance for the standard flushing method is then

$$
R_{\mathrm{ST}}=R_{\text {Post }}+R_{\text {Wall }}+R_{\text {Field }} \text {. }
$$

The total hydraulic resistance for hydrodynamic flushing is

$R_{\mathrm{HY}}=\left(R_{\text {Post }}+R_{\text {Wall }}+R_{\text {Field }}\right) / / R_{\mathrm{TH} 1} / /\left(R_{\mathrm{TH} 2-4}+2 R_{\text {Post }}\right)$,

where $R_{\mathrm{TH} 1-4}$ represent the four through-hole fluidic channels. Using the dimensions stated in table 2 and using $m=1-10^{5}$, the analytically estimated resistances are $R_{\mathrm{Pos} t}=6.96 \times 10^{12}$, $R_{\text {Wall }}=4.39 \times 10^{12}, R_{\text {Field }}=6.50 \times 10^{9}$ and $R_{\mathrm{TH}}=2.31 \times 10^{11}$ $(\mathrm{Pa} \mathrm{s}) \mathrm{m}^{-3}$. This gives $R_{\mathrm{ST}}=1.14 \times 10^{13}$ and $R_{\mathrm{HY}}=2.16 \times$ $10^{11}$, a $53 \times$ reduction in steady state hydraulic resistance.

\subsection{Results}

The enclosed perimeter patterns were machined into the workpiece as deep as possible. The standard flushing pattern stalled indefinitely at a tool plunge depth of $41.3 \mu \mathrm{m}$ due to debris accumulation. The hydrodynamic pattern plunged $125 \mu \mathrm{m}$ for the through-holes and then $80 \mu \mathrm{m}$ for the walled structure before being stopped manually. During the second step, the bubbles generated during machining coalesced almost exclusively within the wall perimeter. Very few bubbles were visible escaping out the sides. As the bubbles rose up the through-holes, they entrained debris particles and flushed them out. Bubble size is important because in fluidization column systems, the larger the gas bubbles, the more efficient the solid particle removal $[22,30]$.

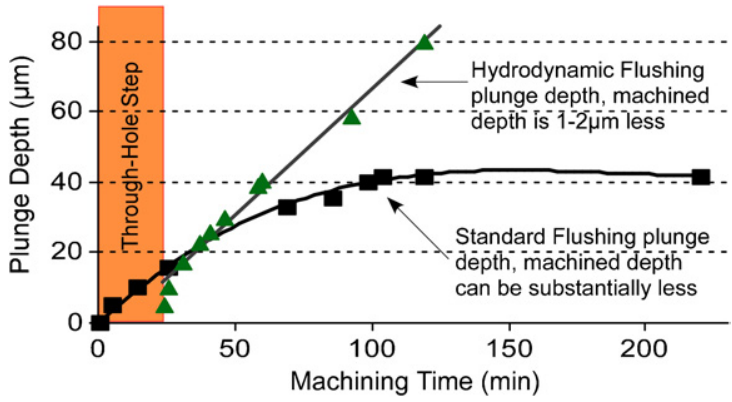

Figure 13. Plunge depth versus machining time for standard flushing and two-step hydrodynamic flushing. The standard walled pattern self-terminates at $40 \mu \mathrm{m}$. The two-step process required an initial through-hole step but eventually surpassed the plunge depth of the standard case and maintained a linear trend for each step.

SEM images of the machined workpiece and tools show that the two-step procedure had a dramatic impact on dimensional tolerance, sidewall angle, surface finish and device height (figure 12). The original grain structure is still visible on the surface of the workpiece.

Figure 13 plots the plunge depth over time for the two flushing methods. Despite machining twice as deep, the twostep flushing method progressed much faster. According to the model in section $\mathrm{B}$, the hydrodynamic hydraulic resistance $R_{\mathrm{HY}}$ decreases with increasing depth while the standard flushing resistance $R_{\mathrm{ST}}$ increases with increasing depth. The standard method follows a third power trend while the two-step hydrodynamic method follows a linear trend for both steps. 


\section{Discussion and conclusion}

The integration of lithographically patterned tools with $\mu \mathrm{EDM}$ has the potential to remove a major throughput bottleneck in achieving precision micromachined patterns in bulk metal foils. However, as features are spaced closer together, debris accumulation and gas trapping become very important design considerations due to a corresponding increase in spurious discharges. A batch mode $\mu \mathrm{EDM}$ parametric study on the effects of debris accumulation in commonly used lithography patterns was presented in this work to illuminate the design space. It was found that $10 \mu \mathrm{m}$ lines required the lowest machining tolerance to produce a desired pattern with a tradeoff on tool wear, but the $25 \mu \mathrm{m}$ lines provide a more reasonable compromise. Careful consideration should be taken with regard to compensating for tool wear around intersections and corners. Simulations of the hydraulic resistance for isolated line features indicate that debris is more likely to escape along the corners. The increased discharge gap in the center of large patterns should also be compensated. This study provides motivation for developing new debris mitigation techniques that take advantage of the lithographic processes available during tool fabrication.

A new passivated tool electrode process for batch mode $\mu \mathrm{EDM}$ was investigated. It is easily integrated with any die sinker or serial-type electrical machining process to significantly reduce spurious discharges. This could include machining techniques outside of $\mu \mathrm{EDM}$ such as electrochemical discharge machining. While machining time increased due to debris accumulation, mushroom-shaped recasting was eliminated and tool wear was negligible. The workpiece machined with the coated tool had significantly better feature and edge definition, smoother surfaces, was deeper, and more uniform than the uncoated workpiece. The test patterns were $4.5 \mu \mathrm{m}$ wide, $25 \mu \mathrm{m}$ tall and $1 \mathrm{~mm}$ long unreleased stainless steel beams. A silicon passivation coating provides one component of a high density batch $\mu \mathrm{EDM}$ solution. It extends the usable life of a tool, but does not eliminate the debris. When combined with a mechanism for debris flushing, machining time drops dramatically, allowing for deep, high density, batch $\mu \mathrm{EDM}$.

Debris accumulation is a problem not only for high fill factor patterns, but also for enclosed patterns with smaller fill factors. A hydrodynamic flushing method utilizing selfgenerated bubbles for debris entrainment was investigated. A rough hydraulic resistance estimate obtained using a static uniaxial model predicted a $53 \times$ reduction with the new method. Significant improvements in surface and edge finish as well as machining time and depth were observed. The technique does require a pattern that allows through-holes to be machined first and also therefore a tool height exceeding the thickness of the workpiece. More die area is required, but if the location of the through-holes is kept constant, the through-hole pattern dies could be reused many times.

\section{Acknowledgments}

The authors would like to thank Dr Kenichi Takahata and Dawn S Skala for discussions. This work was supported in part by Sandia National Laboratories, the Engineering Research Centers Program of the National Science Foundation under award number EEC-9986866 and the University of Michigan. The facilities used for this research include the Michigan Nanofabrication Facility (MNF) at the University of Michigan. The Advanced Light Source is supported by the Mat. Sci. Div., of the U.S. Department of Energy (DOE) under contract DE-AC03-76SF00098 at Lawrence Berkeley National Laboratory. Sandia is operated by Sandia Corp. for the DOE's Nat. Nuclear Security Admin. under contract DE-AC04-94AL85000.

\section{References}

[1] Masaki T, Kawata K and Masuzawa T 1990 Micro electro-discharge machining and its applications Proc. of IEEE MEMS 1990 pp 21-6

[2] Ho K H and Newman S T 2003 State of the art electrical discharge machining (EDM) Int. J. Mach. Tools Manuf. 43 1287-300

[3] Ehrfeld W, Lehr H, Michel F and Wolf A 1996 Microelectro discharge machining as a technology in micromachining SPIE's Symp. Micromachining and Microfabrication vol 2879, pp 332-7

[4] Koch O, Ehrfeld W and Michel F 2000 Micro-electro discharge machining-application, strategy and technology Proc. 2 nd Int. Workshop Microfactories (IWMF) pp 79-82

[5] Takahata K and Gianchandani Y B 2002 Batch mode micro-electro-discharge machining J. MEMS 11 102-10

[6] Takahata K, DeHennis A, Wise K D and Gianchandani Y B 2003 Stentenna: a micromachined antenna stent for wireless monitoring of implantable microsensors IEEE Conf. EMBS pp 3360-3

[7] Stampfl J, Leitgeb R, Cheng Y-L and Prinz F B 2000 Electro-discharge machining of mesoscopic parts with electroplated copper and hot-pressed silver tungsten electrodes J. Micromech. Microeng 10 1-6

[8] Amorim F L and Weingaertner W L 2004 Die-sinking electrical discharge machining, of a high-strength copper-based alloy for injection molds J. Braz. Soc. Mech. Sci. Eng 26 137-44

[9] Udeshi K, Richardson M, Hung J-J, Que L, Rebeiz G M and Gianchandani Y B 2005 A dual-EDM reverse damascene process for RF switches and other bulk devices Proc. of IMECE, MEMS Div. pp 137-41

[10] Takahata K 2005 Batch manufacturing technology based on micro-electro-discharge machining and application to cardiovascular stents PhD Thesis University of Michigan

[11] McGeough J A 1998 Advanced Methods of Machining (New York: Chapman and Hall) pp 128-52

[12] Brown J 1998 Advanced Machining Technology Handbook (New York: McGraw Hill)

[13] El-Hofy H 2005 Advanced Machining Processes. Nontraditional and Hybrid Machining Processes (New York: McGraw Hill)

[14] Chapman W 2002 Modern Machine Shop's Handbook for the Metalworking Industries (Cincinatti, OH: Hanser Gardner)

[15] Schumacher B M 2004 After 60 years of EDM the discharge process remains still disputed J. Mater. Process. Technol. $149376-81$

[16] Lewis T J 2003 Breakdown initiating mechanisms at electrode interfaces in liquids IEEE Trans. Dielectr. Electr. Insul. 10 948-54

[17] Schulze H P, Lauter M, Wollenberg G, Storr M and Rehbein W 2001 Investigation of the pre-ignition stage in EDM Proc. 13th Int. Symp. on Electromachining (May 2001) pp 141-52 
[18] Descoeudres A 2006 Characterization of electrical discharge machining plasmas $P h D$ Thesis EPFL

[19] Albinksi K, Musiol K, Miernikiewicz A, Labuz S and Malota M 1996 The temperature of a plasma used in electrical discharge machining Plasma Sources Sci. Tech $5736-42$

[20] Pompili M, Mazzetti C and Forster E O 1992 Partial discharge distributions in liquid dielectrics IEEE Trans. Electr. Insul. 27 99-105

[21] Murphy E J 1970 The generation of gases in the dielectric breakdown of oil J. Phys. D: Appl. Phys. 3 917-23

[22] Kaneko T and Tsuchiya M 1997 Observation of behavior of machining products in die-sinking EDM by using high-speed VTR Int. J. Electr. Mach. 2 37-42

[23] Richardson M T and Gianchandani Y B 2005 A passivated electrode batch $\mu$ EDM technology for bulk metal transducers and packages Proc. IEEE Sensors 219-22

[24] Richardson M T, Gianchandani Y B and Skala D S 2006 A parametric study of dimensional tolerance and hydrodynamic debris removal in micro-electro-discharge machining Proc. IEEE MEMS pp 314-7

[25] L Kao Y, Tu G C, Huang C A and Chang J H 2004 The annealing behaviour of copper deposit electroplated in sulfuric acid bath with various concentrations of thiourea Mater. Sci.Eng. A 382 104-11

[26] Yu Z Y, Rajurkar K P and Shen H 2002 High aspect ratio and complex shaped blind micro holes by micro EDM Ann. CIRP 50 359-62

[27] Zhao Y, Zhang X, Liu X and Yamazaki K 2004 Geometric modeling of the linear motor driven electrical discharge machine (EDM) die-sinking process Int. J. Mach. Tools Manuf. 44 1-9

[28] Kaneko Y, Yamada H, Toyonaga T and Shoda K 2000 Performance of linear motor equipped die-sinking EDM Int. J. Mach. Tools Manuf. 5 59-64

[29] Lim H S, Wong Y S, Rahman M and Edwin Lee M K 2003 A study on the machining of high-aspect ratio micro-structures using micro-EDM J. Mater. Process. Technol $140318-25$
[30] Yeo S H and Tan L K 1999 Effects of ultrasonic vibrations in micro-electro-discharge machining of microholes J. Micromech. Microeng. 9 345-52

[31] Zhang Q H, Zhang J H, Ren S F, Deng J X and Ai X 2004 Study on technology of ultrasonic vibration aided electrical discharge machining in gas J. Mater. Process. Technol 149 640-4

[32] Gao C and Liu Z 2003 A study of ultrasonically aided micro-electrical-discharge machining by the application of workpiece vibration J. Mater. Process. Technol. 139 226-8

[33] Yeo S H and Tan L K 1999 Effects of ultrasonic vibrations in micro electro-discharge machining of microholes J. Micromech. Microeng. $9345-52$

[34] Kumagai S, Misawa N, Takeda K and Abdukarimov E T 2003 Plasma-applied machining of a narrow and deep hole in a metal using a dielectric-encased wire electrode Thin Solid Films 457 180-5

[35] Aoyama S, Tamura K, Sato T, Kimura T, Sawahata K and Nagai T 1999 High-performance coated wire electrodes for high-speed cutting and accurate machining Hitachi Cable Rev. 18 75-80

[36] Masuzawa T, Fujino M, Kobayashi K and Suzuki T 1985 Wire electro-discharge grinding for micromachining CIRP Ann. 34 431-4

[37] Morf W E, Guenat O T and de Rooij N F 2001 Electroosmotic pumping in complex capillary systems: 1 . Principles and general theoretical approach Sensors Actuators B 72 266-72

[38] Tobazeon R 1996 Electrohydrodynamic behaviour of single spherical or cylindrical conducting particles in an insulating liquid subjected to a uniform DC field J. Phys. D: Appl. Phys 29 2595-608

[39] Soni J S 1994 Microanalysis of debris formed during rotary EDM of titanium alloy (Ti 6Al 4V) and die steel (T 215 Cr12) Wear J. 177 71-9

[40] Bokhonov B and Korchagin M 2002 In-situ investigation of the formation of eutectic alloys in the systems silicon-silver and silicon-copper J. Alloys Compd. 335 149-56 